\title{
Design Considerations and Analysis of Potential Applications of a High Power Ultraviolet FEL at the TESLA Test Facility at DESY
}

\author{
C. Pagani ${ }^{\mathrm{a}}$, E.L. Saldin ${ }^{\mathrm{b}}$, E.A. Schneidmiller ${ }^{\mathrm{b}}$ and M.V. Yurkov ${ }^{\mathrm{c}}$ \\ a INFN Milano - LASA, Via Cervi, 201, 20090 Segrate (MI), Italy \\ ${ }^{b}$ Automatic Systems Corporation, 443050 Samara, Russia \\ ${ }^{\mathrm{C}}$ Joint Institute for Nuclear Research, Dubna, 141980 Moscow Region, Russia
}

\begin{abstract}
This paper presents analysis of a possibility of constructing a high power ultraviolet free electron laser at the TESLA Test Facility at DESY. Proposed facility consists of a tunable master oscillator $\left(P_{\mathrm{av}} \sim 10 \mathrm{~mW}, P_{\text {peak }} \sim 10 \mathrm{~kW}, \lambda \simeq 200-350 \mathrm{~nm}\right)$ and FEL amplifier with tapered undulator. The average and the peak radiation power at the exit of the FEL amplifier are about $7 \mathrm{~kW}$ and $220 \mathrm{GW}$, respectively. Installation of such a facility can extend significantly scientific potential of the TESLA Test Facility. UV free electron laser can be used for constructing a polarized, monochromatic gamma-source with ultimate yield up to $10^{12}$ gamma-quanta per second with maximal energy about $100 \mathrm{MeV}$. Intensive gamma-source can also form the base for constructing the test facility for the TESLA positron generation system. Another accelerator application of the proposed facility is verification of the main technical solutions for the laser and the optical system to be used in the gamma-gamma option of the TESLA collider. Also, high average power UV laser has perspective for industrial applications.
\end{abstract}




\section{Introduction}

A $1 \mathrm{GeV}$ superconducting linear accelerator being under construction at the TESLA Test Facility (TTF) at DESY will produce electron beam with high average and peak power, low energy spread and emittance. The main practical application of this accelerator is to use it for driving the soft X-ray free electron laser [1, 2]. The scope of practical applications of the TESLA Test Facility can be extended significantly with installation of additional free electron laser operating at a longer wavelength, around $200-350 \mathrm{~nm}$, but with much higher average power, up to $7 \mathrm{~kW}$.

In this paper we perform design consideration of a high power UV free electron laser at the Tesla Test Facility. It is supposed to install an additional FEL beamline in the same tunnel in parallel with the soft X-ray FEL. UV FEL will use the same electron beam as an X-ray FEL, thus providing minimal interference between these two options. It will be constructed as a Master Oscillator Power Amplifier (MOPA) scheme. Radiation from a quantum laser $\left(P_{\mathrm{av}} \sim 10 \mathrm{~mW}, P_{\text {peak }} \sim 10 \mathrm{~kW}, \lambda \simeq 200-350 \mathrm{~nm}\right.$ ) will be amplified in the FEL amplifier with a tapered undulator providing peak and average output power up to $220 \mathrm{GW}$ and $7 \mathrm{~kW}$, respectively.

The laser with such ultimate parameters (tunability, high peak and average power) is unique device itself and definitely will attract scientists from different fields of research. In this paper we study in detail a possibility of constructing a high intensity, polarized, monochromatic gamma-source with ultimate yield of gamma-quanta up to $10^{12}$ per second. This value exceeds by five orders of magnitude parameters of the best gamma-sources used for nuclear physics experiments at present.

One of the main problems to be solved prior construction of the TESLA collider is that of the positron source. At present it is assumed to produce positrons in a thin target by means of conversion gamma-quanta. Energy spectrum and temporal characteristics of the proposed gamma-source are similar to those to be used in the TESLA project. Estimations presented in the paper show that the yield of positrons from a target irradiated by the gamma-beam will be about $5 \times 10^{12} \mathrm{~s}^{-1}$. This value is high enough to perform the testing of all key systems of the positron production system. It is shown also that the proposed facility reveals unique possibility for experimental verification of a polarized positron source for linear collider.

In the case of the gamma-gamma collider at TESLA there is no reliable technical solution for a conventional laser system with required parameters, and free electron laser system is considered now as a candidate for the laser. Parameters of the high power UV FEL at the TESLA Test Facility and of the optical system of the gamma-source are very close to those used in the project of the gamma-gamma collider. Successful realization 
Table 1. Parameters of the TTF accelerator

\begin{tabular}{ll}
\hline Energy & $1 \mathrm{GeV}$ \\
Mode of operation & quasi-CW \\
Repetition rate & $10 \mathrm{pps}$ \\
Macropulse duration & $800 \mu \mathrm{s}$ \\
Number of bunches per macropulse & $7200 / 14400$ \\
Micropulse repetition rate & $9 \mathrm{MHz} / 18 \mathrm{MHz}$ \\
Charge per bunch & $1 \mathrm{nC}$ \\
Average power in the electron beam & $72 \mathrm{~kW} / 144 \mathrm{~kW}$ \\
Peak current & $2.5 \mathrm{kA}$ \\
Bunch length (RMS) & $50 \mu \mathrm{m}$ \\
Normalized emittance (RMS) & $2 \mathrm{~mm}$ mrad \\
Energy spread (RMS) & $1 \mathrm{MeV}$ \\
\hline
\end{tabular}

of this project will serve as an experimental verification of a technical feasibility of the gamma-gamma option at TESLA.

Finally, the high power ultraviolet laser beam can be used for a variety of industrial applications.

\section{Design considerations for the UV FEL}

Taking into account high quality and high average power of the electron beam from the TTF accelerator it seems to be reasonable to consider a possibility to use this beam for driving a high power FEL amplifier operating in ultraviolet wavelength range. In this paper we consider two options for such a laser. The first one uses untapered undulator of $13 \mathrm{~m}$ length providing the peak and the average output radiation power of $20 \mathrm{GW}$ and $860 \mathrm{~W}$, respectively. The second option assumes to install additional tapered undulator which will allow to increase the peak and the average power up to the values of $220 \mathrm{GW}$ and $7.2 \mathrm{~kW}$, respectively. To reduce interference with the soft X-ray FEL, it is assumed to use the electron beam with the same parameters as for X-ray option (energy, current, time structure, etc.). It seems to be reasonable to place the UV FEL undulator in the same tunnel in parallel with the soft X-ray undulator (see Fig. 1).

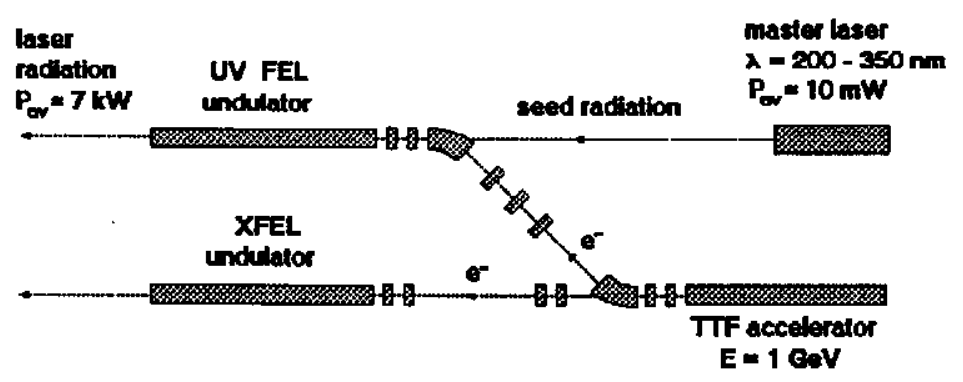

Fig. 1. Scheme of the UV FEL at the TESLA Test Facility

UV FEL facility will be designed as MOPA scheme. Radiation from master oscillator (Nd glass laser or dye laser) of a low peak and average power will be amplified in the FEL amplifier up to the above mentioned values. 
Parameters of the TTF accelerator are presented in Table 1. All the numerical examples presented in the paper correspond to the micropulse repetition rate of the accelerator of $18 \mathrm{MHz}$.

\subsection{Master laser}

In this conceptual design we assume to use conventional quantum generator as master laser. The requirements to the master laser are relatively moderate with respect to the required value of the peak power which should exceed the shot noise power in the electron beam (of about 30 watts in our case). More severe requirement is that the laser time diagram of operation should follow the time diagram of the accelerator. There exist two types of lasers which can be used for this purpose. The most simplest way is to use 4 th or 5 th harmonic of the Nd glass laser $(\lambda=262 \mathrm{~nm}$ and $209 \mathrm{~nm}$ ). In this case it is necessary to install an additional $\mathrm{Nd}$ glass amplifier which will amplify radiation from a master oscillator of the photoinjection system. At the exit of the amplifier the radiation will be converted into 4 th or 5 th harmonic. Peak power of the radiation will be about $5 \mathrm{~kW}$ within $\sim 10$ ps pulse duration. The problem of synchronization of electron and laser pulses will be solved automatically since they use common master oscillator and timing system. Average output power of the master laser for UV FEL is by an order of magnitude less than the output power of existent laser for the photoinjector. When there will be necessary to tune the radiation wavelength, dye laser system pumped by Nd glass laser can be used.

\subsection{FEL amplifier}

Starting point of the design are the project parameters of the electron beam from the TTF accelerator (see Table 1) [1]. Undulator period has been chosen to be equal to $7 \mathrm{~cm}$. At the operating wavelength of the FEL amplifier around $200 \mathrm{~nm}$ the peak value of the magnetic field is about $1 \mathrm{~T}$ for the planar undulator and about $0.7 \mathrm{~T}$ for the helical one. Main physical effects influencing the operation of the FEL amplifier in this region of parameters are the diffraction effects, the space charge effects and the slippage effect. Optimization of the parameters of the FEL amplifier has been performed with nonlinear, three-dimensional, time-dependent simulation code S\&D-3T which takes into account all these effects [3]. The longitudinal profile of the electron bunch assumed to be Gaussian with $\sigma_{\mathrm{z}}=50 \mu \mathrm{m}$. Calculations have been performed for the planar undulator. In the case of the helical undulator the output characteristics will be approximately the same at a shorter undulator length.

FEL amplifier with untapered undulator. We start our study with the case of the FEL amplifier with untapered undulator. The process of amplification starts from the seed 
Table 2. Parameters of the UV FEL with untapered undulator

\begin{tabular}{ll}
\hline Undulator & Planar \\
\hline Type & $7 \mathrm{~cm}$ \\
Period & $0.99 \mathrm{~T}$ \\
Maximal field & $13 \mathrm{~m}$ \\
Length & $83 \mu \mathrm{m}$ \\
Beam size in the undulator (RMS) & \\
Radiation & $200-350 \mathrm{~nm}$ \\
Wavelength & Diffraction limited \\
Dispersion & $140 \mu \mathrm{m}$ \\
Spot size at the undulator exit (HWHM) & $0.18 \mathrm{mrad}$ \\
Angular divergence (HWHM) & $250 \mathrm{fs}$ \\
Pulse duration (FWHM) & $10 \mathrm{pps}$ \\
Repetition rate & $800 \mu \mathrm{s}$ \\
Macropulse duration & 14400 \\
Number of pulses per macropulse & $18 \mathrm{MHz}$ \\
Micropulse repetition rate & $20 \mathrm{GW}$ \\
Peak output power & $860 \mathrm{~W}$ \\
Average power & $0.6 \%$ \\
Efficiency & \\
\hline
\end{tabular}

signal of the master laser with peak power of $5 \mathrm{~kW}$. Saturation power and saturation length depend on the tuning of the FEL amplifier. In Fig. 2 we present the dependence on the wavelength deviation from exact FEL resonance of the energy in the radiation pulse for different lengths of the undulator. It can be simply rescaled to the case of the energy deviation from resonance value as $\Delta \mathcal{E} / \mathcal{E}=\Delta \lambda / 2 \lambda$. It is seen from this plot that the energy in the radiation pulse can be increased by an increase of the undulator length and an appropriate tuning of the FEL amplifier parameters (by means of tuning the seed signal frequency, or the energy of the electron beam). We fix our choice with the length of the undulator of $13 \mathrm{~m}$. General parameters of the UV FEL with untapered undulator operating at the saturation are presented in Table 2. Total energy in the radiation pulse at the undulator exit is equal to $6 \mathrm{~mJ}$, pulse duration is about $250 \mathrm{fs}$, and the peak and the average power are about $20 \mathrm{GW}$ and $860 \mathrm{~W}$, respectively (see Fig. 3). Radial distribution of the radiation intensity at the undulator exit and the directivity diagram of the output radiation in far zone are shown in Figs. 4 and 5. Energy spread of the electrons at the exit of the undulator is about $1 \%$ (see Fig. 6).

Fluctuations of the electron beam and the master laser parameters (energy, charge in the bunch, power of the master laser) will lead to the fluctuations of the output power of the FEL amplifier. Sensitivity of the FEL amplifier operation to the fluctuations of the energy of the electron beam can be obtained form the plots presented in Fig. 2 (by means of recalculating the wavelength deviation into the energy deviation as $\Delta \mathcal{E} / \mathcal{E}=\Delta \lambda / 2 \lambda$ ). Sensitivity of the FEL amplifier operation to the fluctuations of the bunch charge and to the fluctuations of the input laser power is illustrated with Figs. 7 and 8. Expected relative fluctuations have the following values: of about $0.1 \%$ for the electron energy, of about $10 \%$ for the bunch charge, and of about $10 \%$ for the input radiation power. Analysis of 

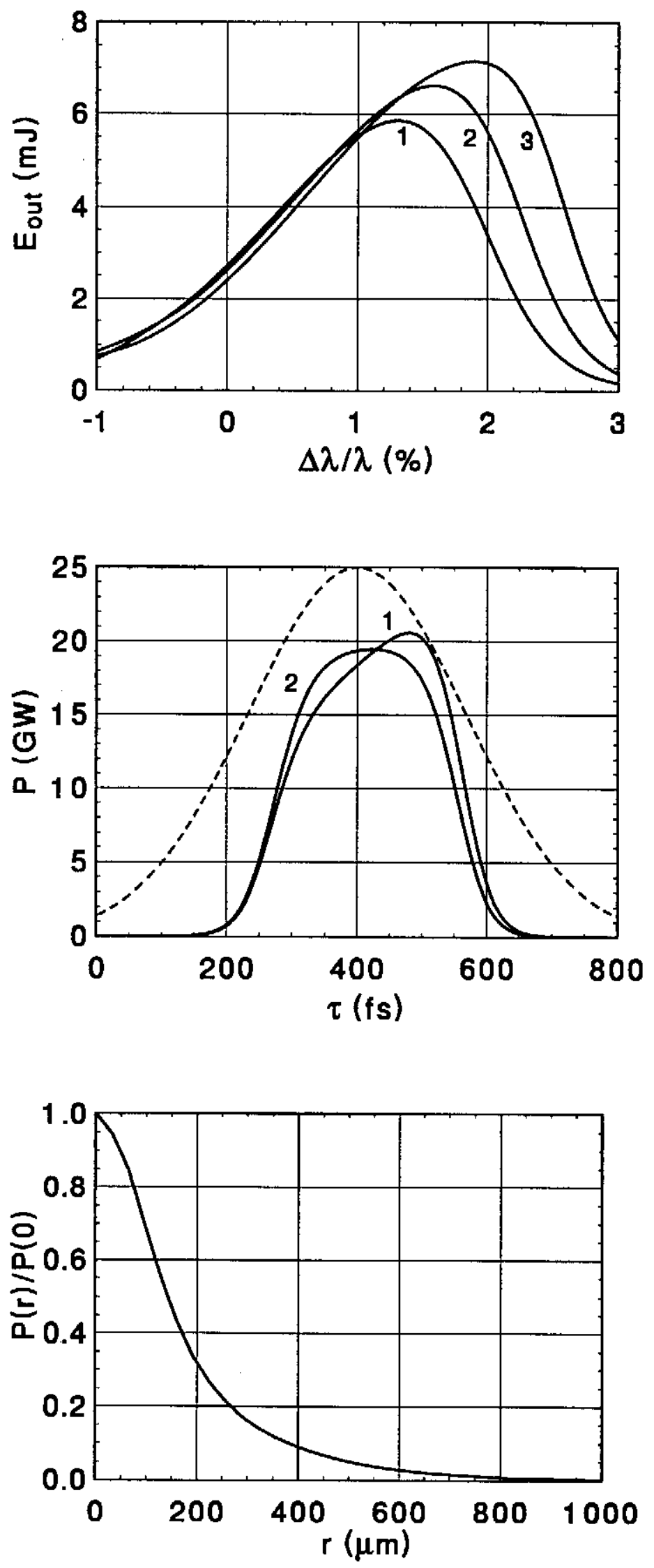

Fig. 2. Dependence on the wavelength deviation from exact FEL resonance of the energy in the radiation pulse produced by the FEL amplifier with untapered undulator. Curves 1, 2 and 3 correspond to the undulator length of $13 \mathrm{~m}, 14 \mathrm{~m}$ and $15 \mathrm{~m}$, respectively
Fig. 3. Time structure of the radiation pulse produced by the FEL amplifier with untapered undulator (curve 1) and of the power loss in the electron bunch (curve 2). Dashed line is the longitudinal profile of the electron bunch (maximal value of the beam current is equal to $2.5 \mathrm{kA}$ )
Fig. 4. Radial distribution of the radiation intensity at the undulator exit for the FEL amplifier with untapered undulator operating at the saturation 

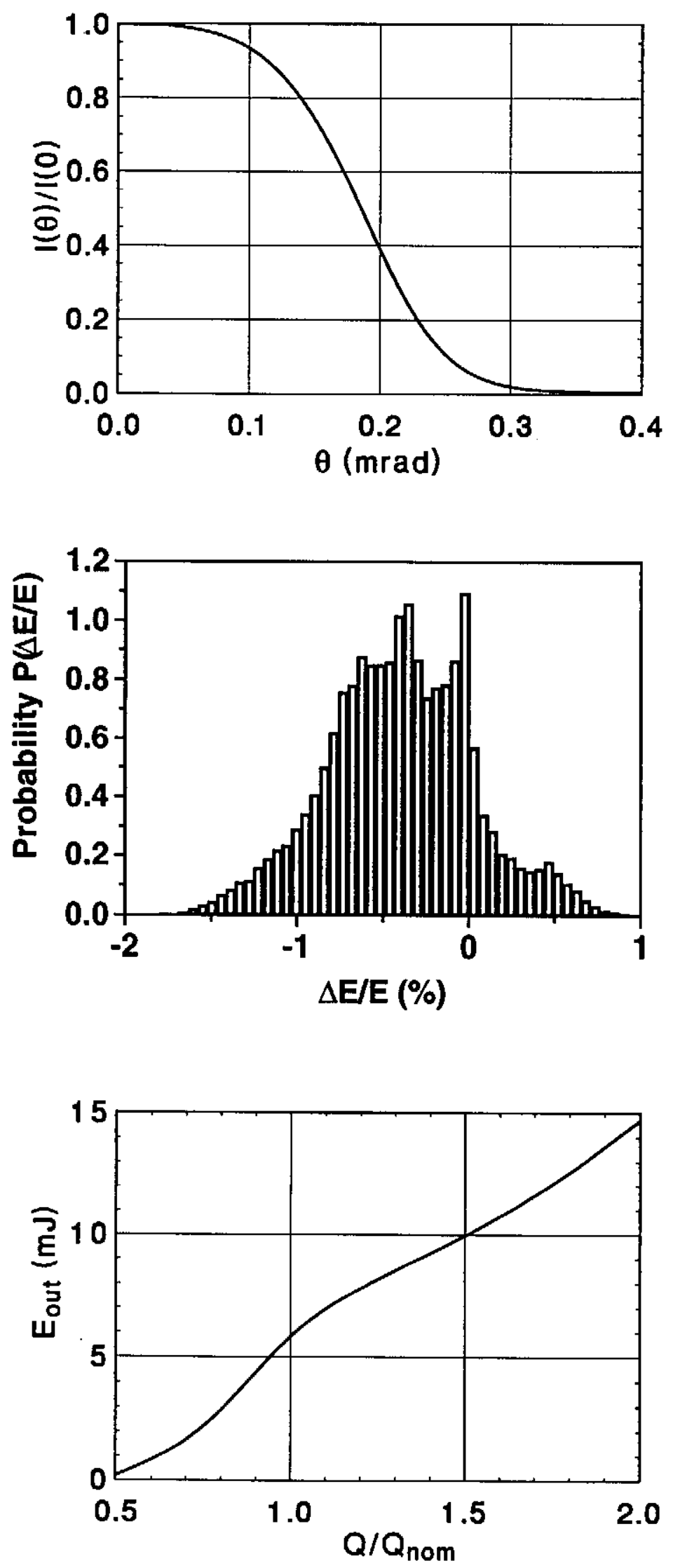

Fig. 5. Directivity diagram of the radiation intensity in far zone for the FEL amplifier with untapered undulator operating at the saturation
Fig. 6. Energy distribution of the electrons at the exit of the FEL amplifier with untapered undulator operating at the saturation

Fig. 7. Dependence of the energy in the radiation pulse on the bunch charge for the FEL amplifier with untapered undulator 


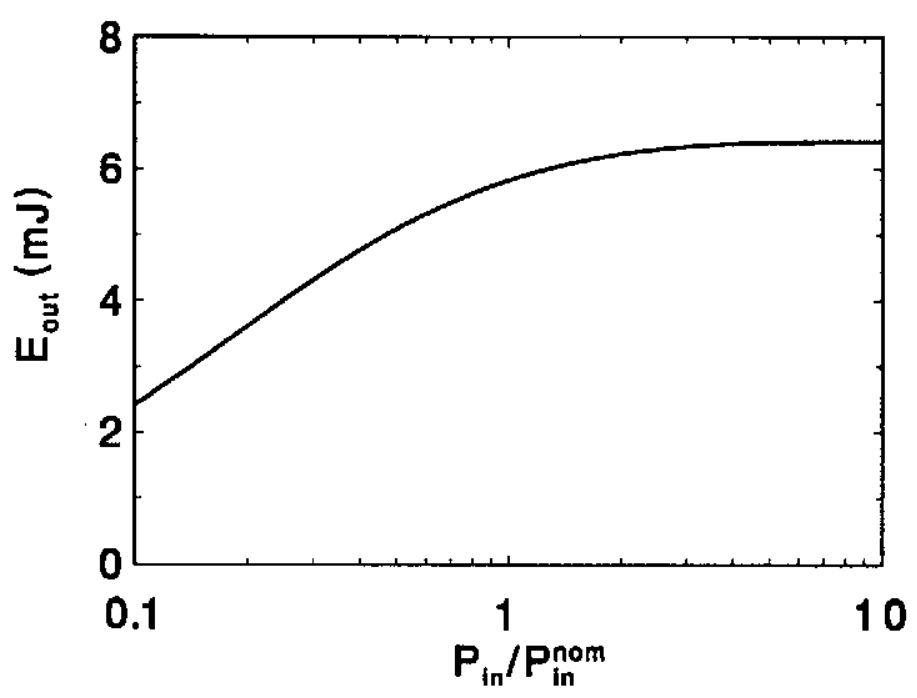

Fig. 8. Dependence of the energy in the radiation pulse on the input radiation power for the FEL amplifier with untapered undulator

the output characteristics of the FEL amplifier shows that it will operate reliably within significant safety margin at expected fluctuations of the electron and input laser beam.

High power FEL amplifier with tapered undulator. Saturation of the radiation power in the FEL amplifier occurs due to the energy losses by the particles which fall out of the resonance with the electromagnetic wave. Nevertheless, effective amplification of the radiation is possible in the nonlinear regime by means of using tapered undulator $[5,6,7]$. It has been demonstrated in the microwave FEL amplifier with extraction efficiency $34 \%$ [8]. In principle, high efficiency can be also achieved in the FEL amplifier operating in the visible or UV wavelength range. There are no principal physical limitations - the larger undulator length, the higher efficiency can be achieved at an appropriate undulator tapering [4]. Calculations based on the steady-state theory shows that at the length of the undulator of $30 \mathrm{~m}$, the efficiency of the UV FEL amplifier at the TESLA Test Facility can reach the value of $10 \%$. Nevertheless, at such a length of the undulator one should take into account the slippage effect. For instance, at the undulator period equal to $7 \mathrm{~cm}$ and the radiation wavelength equal to $200 \mathrm{~nm}$, kinematic slippage of the radiation with respect to the electron bunch is about $90 \mu \mathrm{m}$ which is comparable with the length of the electron bunch, $2 \sigma_{\mathrm{z}} \simeq 100 \mu \mathrm{m}$. So, steady-state approach can be used only for rough estimations, and parameters of the FEL amplifier should be optimized using time-dependent approach.

Optimized parameters of the FEL amplifier are presented in Table 3. The length of untapered section of the undulator is equal to $12 \mathrm{~m}$. The field in the tapered section is reduced by a linear law from $0.99 \mathrm{~T}$ down to the value of $0.87 \mathrm{~T}$ at the end of the undulator of $28 \mathrm{~m}$. Figure 9 presents evolution along the undulator length of the energy in the radiation pulse. In Fig. 10 we present the time structure of the radiation pulse at the exit of the undulator. We obtain that the peak radiation power of about $220 \mathrm{GW}$ is close to that predicted by the steady-state calculations. The duration of the radiation 
Table 3. Parameters of the UV FEL with tapered undulator (high power option)

\begin{tabular}{ll}
\hline Undulator & \\
\hline Type & Planar \\
Period & $7 \mathrm{~cm}$ \\
Maximal field (entr./exit) & $0.99 \mathrm{~T} / 0.87 \mathrm{~T}$ \\
Total length & $28 \mathrm{~m}$ \\
Length of untapered section & $12 \mathrm{~m}$ \\
Beam size in the undulator (RMS) & $83 \mu \mathrm{m}$ \\
Radiation & \\
Wavelength & $200-350 \mathrm{~nm}$ \\
Dispersion & Diffraction limited \\
Spot size at the undulator exit (HWHM) & $170 \mu \mathrm{m}$ \\
Angular divergence (HWHM) & $0.13 \mathrm{mrad}$ \\
Pulse duration (FWHM) & $230 \mathrm{fs}$ \\
Repetition rate & $10 \mathrm{pps}$ \\
Macropulse duration & $800 \mu \mathrm{s}$ \\
Number of pulses per macropulse & 14400 \\
Micropulse repetition rate & $18 \mathrm{MHz}$ \\
Peak output power & $220 \mathrm{GW}$ \\
Average power & $7.2 \mathrm{~kW}$ \\
Efficiency & $5 \%$ \\
\hline
\end{tabular}

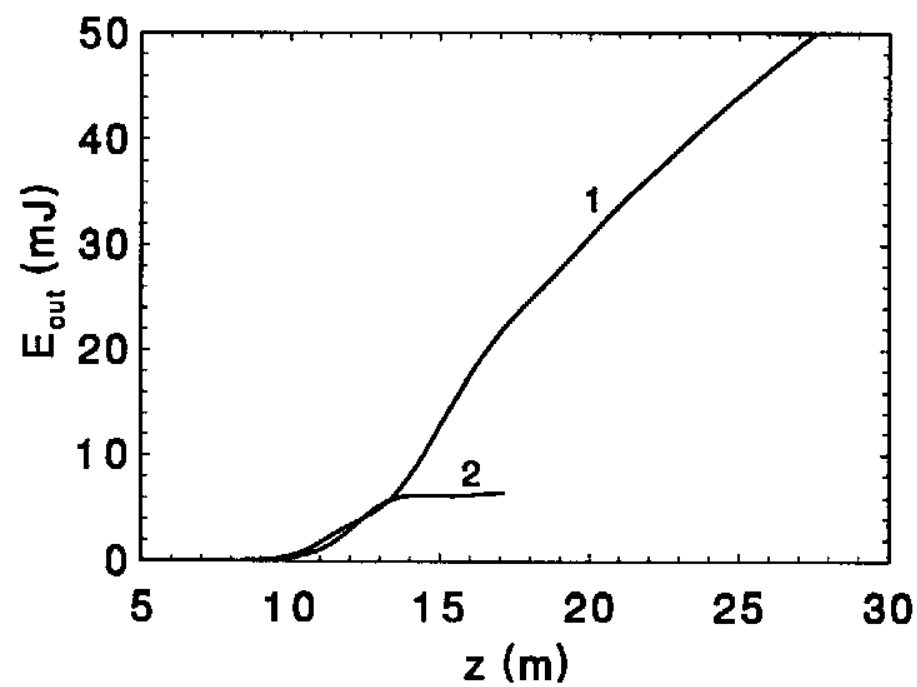

Fig. 9. Energy in the radiation pulse versus the length for the FEL amplifier with tapered undulator (curve 1) and for the FEL amplifier with untapered undulator (curve 2)

pulse is about $230 \mathrm{fs}$. The energy in the radiation pulse is equal to $50 \mathrm{~mJ}$ and conversion efficiency (ratio of the energy in the radiation pulse to the energy in the electron pulse) is equal to $5 \%$. Average power of the radiation from the FEL amplifier with tapered parameters is equal to $7.2 \mathrm{~kW}$. Analysis of the distribution of the beam power losses along the electron beam (see Fig. 10) shows that the radiation is produced mainly by the front fraction of the electron pulse which is a consequence of the slippage effect mentioned above. On the other hand, one can see that actual slippage of the radiation pulse (about $30 \mu \mathrm{m}$ ) is significantly less than the kinematic slippage (about $100 \mu \mathrm{m}$ ). This difference is connected with the interaction of the radiation with the electron beam leading to the dynamic change of the phase of the radiation field. As a result, group velocity of the radiation pulse $(\partial \omega / \partial k)$ becomes to be less than the velocity of light. We see that this 
effect reveals the possibility to achieve high extraction efficiency even in the case of a short electron bunch with respect to the kinematic slippage distance.

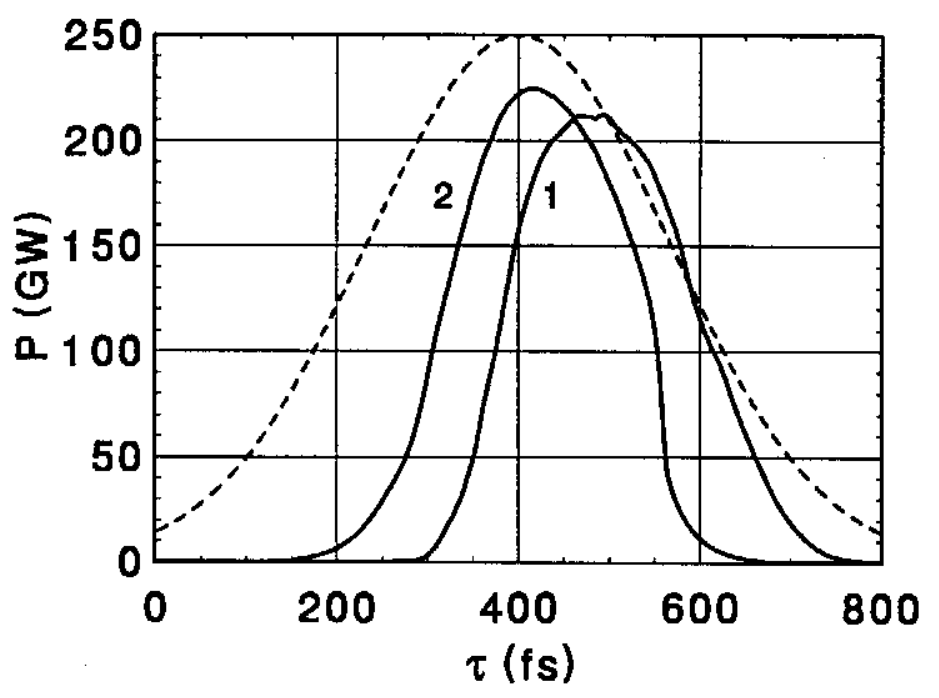

Fig. 10. Time structure of the radiation pulse at the exit of the FEL amplifier with tapered undulator (curve 1) and of the power loss in the electron bunch (curve 2). Dashed line is the longitudinal profile of the electron bunch (maximal value of the beam current is equal to $2.5 \mathrm{kA}$ )

Phase analysis of the particles shows that the electrons of the front part of the beam are well separated into two fractions, of about $70 \%$ of the electrons are trapped in the regime of coherent deceleration (see Fig. 11). The electrons of the back part of the beam fall out the regime of coherent deceleration due to the slippage of the radiation. In Fig. 12 we present energy distribution in the electron beam at the exit of the undulator. It is seen that the energy spectrum of the spent beam is rather wide, of about $12 \%$.

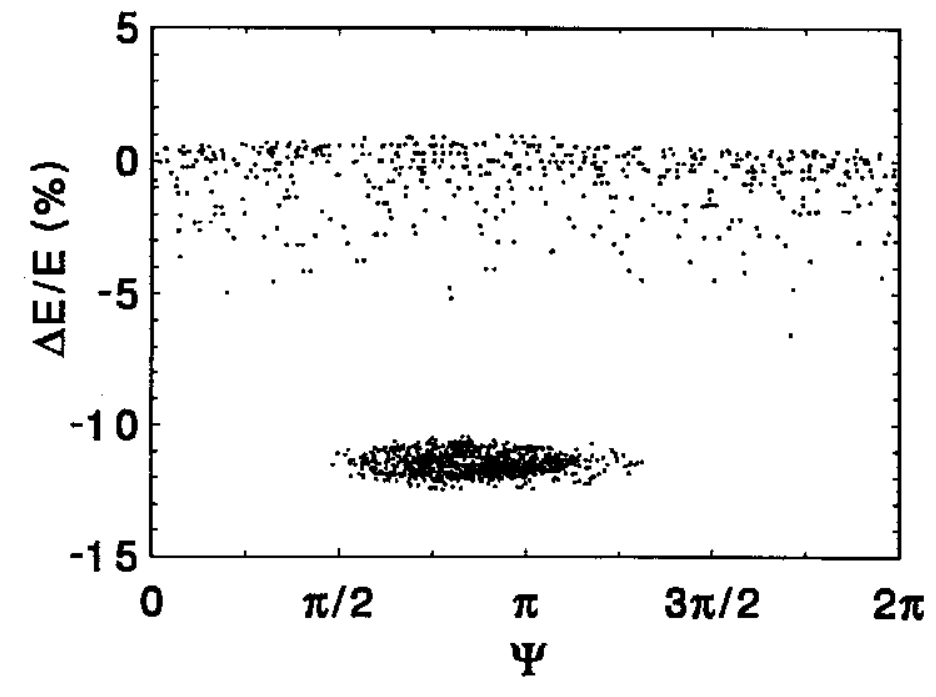

Fig. 11. Phase distribution of the particles in the middle of the electron bunch. Total efficiency of the FEL amplifier is equal to $5 \%$

In Fig. 13 we present radial distribution of the radiation intensity at the exit of the undulator. It is seen that there are long tails spanning up to the distance of $3 \mathrm{~mm}$ apart from the electron beam. This is connected with diffraction effects. In the tapering regime 


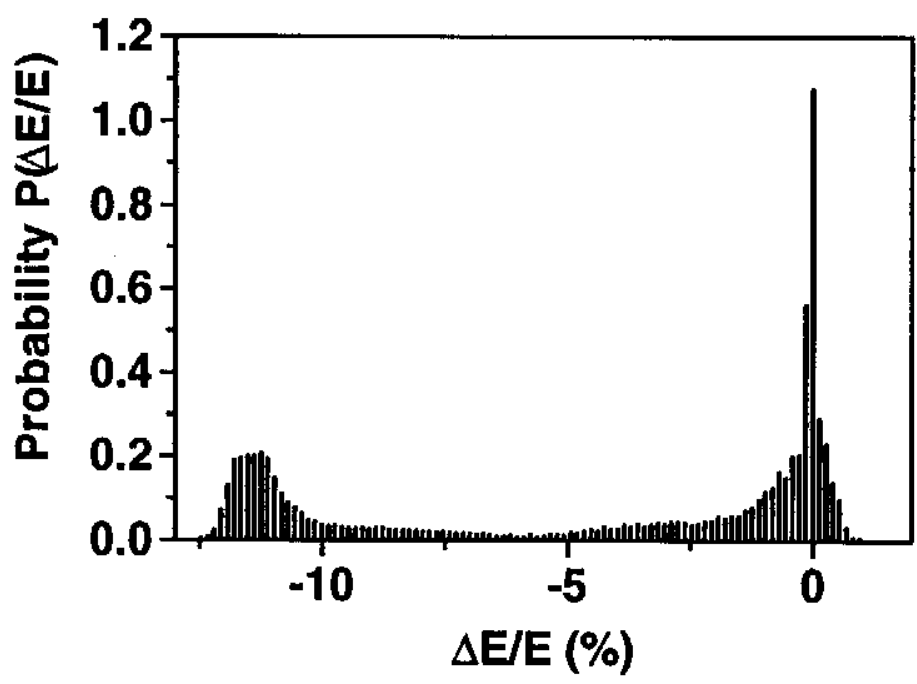

Fig. 12. Energy distribution of the electrons at the exit of the FEL amplifier with tapered undulator. Total efficiency of the FEL amplifier is equal to $5 \%$

the amplitude of the radiation field at the undulator axis almost does not grow, and the radiation field produced by the electron beam continuously expands apart from the source. The larger the tapered section of the undulator, the larger is the diffraction expansion of the radiation. This effect is also reflected on the behaviour of the directivity diagram of the radiation in far diffraction zone (see Fig. 14). It is seen that the width of the angle distribution is visibly smaller than that of the FEL amplifier operating at the saturation (see Fig.5) which is connected with the increasing of the radiation spot size inside the undulator. Also, there are distortions of the directivity diagram connected with complicated shift of the radiation field phase along the transverse coordinate.

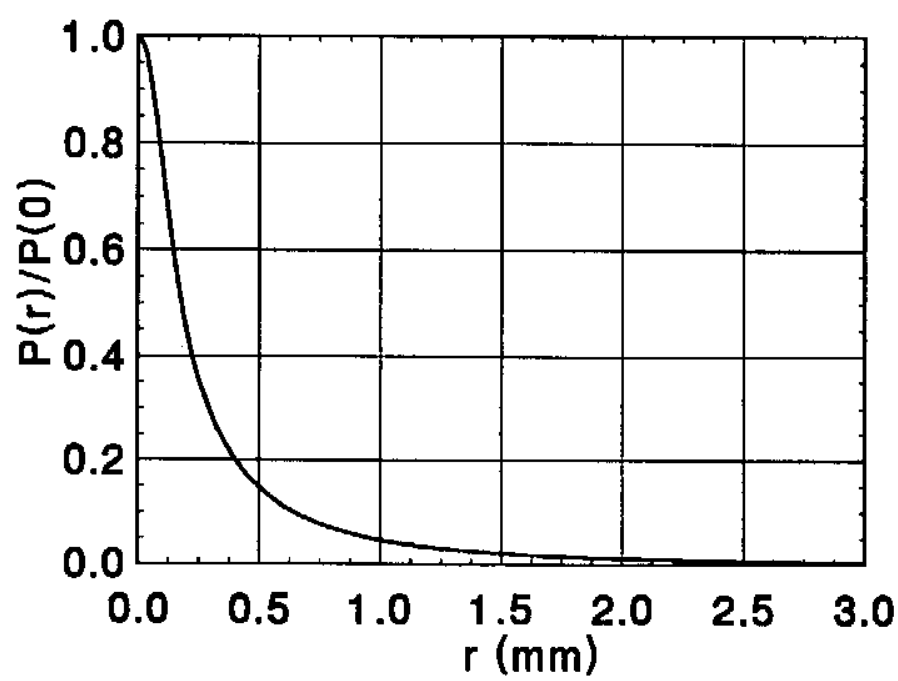

Fig. 13. Radial distribution of the radiation intensity at the undulator exit for the FEL amplifier with tapered undulator. Total efficiency of the FEL amplifier is equal to $5 \%$

The next problem to be studied is the problem of stability of the operation of the FEL amplifier with respect to different jitters (time jitter, energy jitter and jitter of the charge in the electron pulse). First we note that the operation of the FEL amplifier is insensitive 


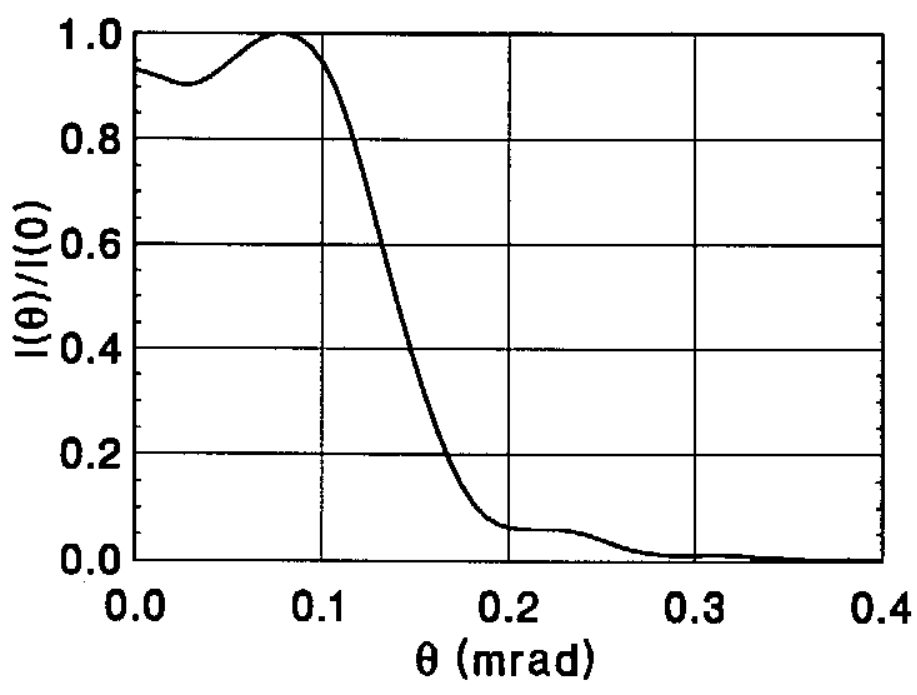

Fig. 14. Directivity diagram of the radiation intensity in far zone for the FEL amplifier with tapered undulator. Total effciency of the FEL amplifier is equal to $5 \%$

to the time jitter, since master laser is triggered by the master oscillator of the injection system. The top of the master laser pulse of duration 10 ps can be easily tuned to the arrival time of a short electron bunch having time jitter of about 1 ps.

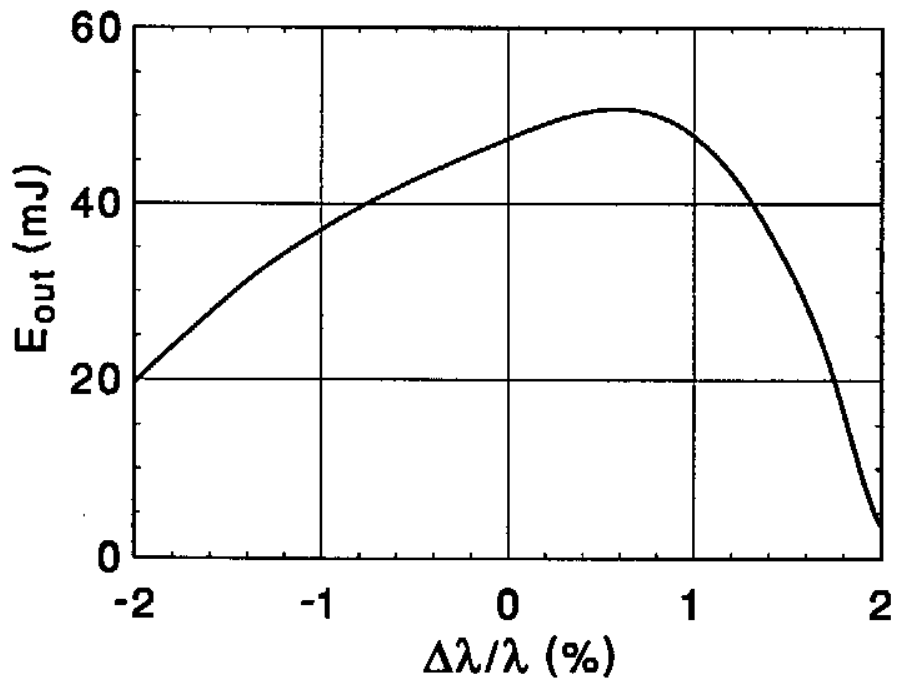

Fig. 15. Dependence on the wavelength deviation from exact FEL resonance of the energy in the radiation pulse of the FEL amplifier with tapered undulator. Total efficiency of the FEL amplifier is equal to $5 \%$

In Fig. 15 we present the dependence of the energy in the radiation pulse as a function of the deviation of the wavelength of the master laser from exact FEL resonance. As it has been mentioned above, this characteristic can be simply rescaled to the case of the energy deviation as $\Delta \mathcal{E} / \mathcal{E}=\Delta \lambda / 2 \lambda$. It is seen that at the designed stability of the energy of the accelerator $(\sim 0.1 \%)$ the fluctuations of the energy in the radiation pulse are about one per cent. The requirement to the stability of the frequency of the master laser is also extremely loose. Sensitivity of the FEL amplifier operation to the fluctuations of the bunch charge and to the fluctuations of the input laser power is illustrated with Figs. 16 and 17. 

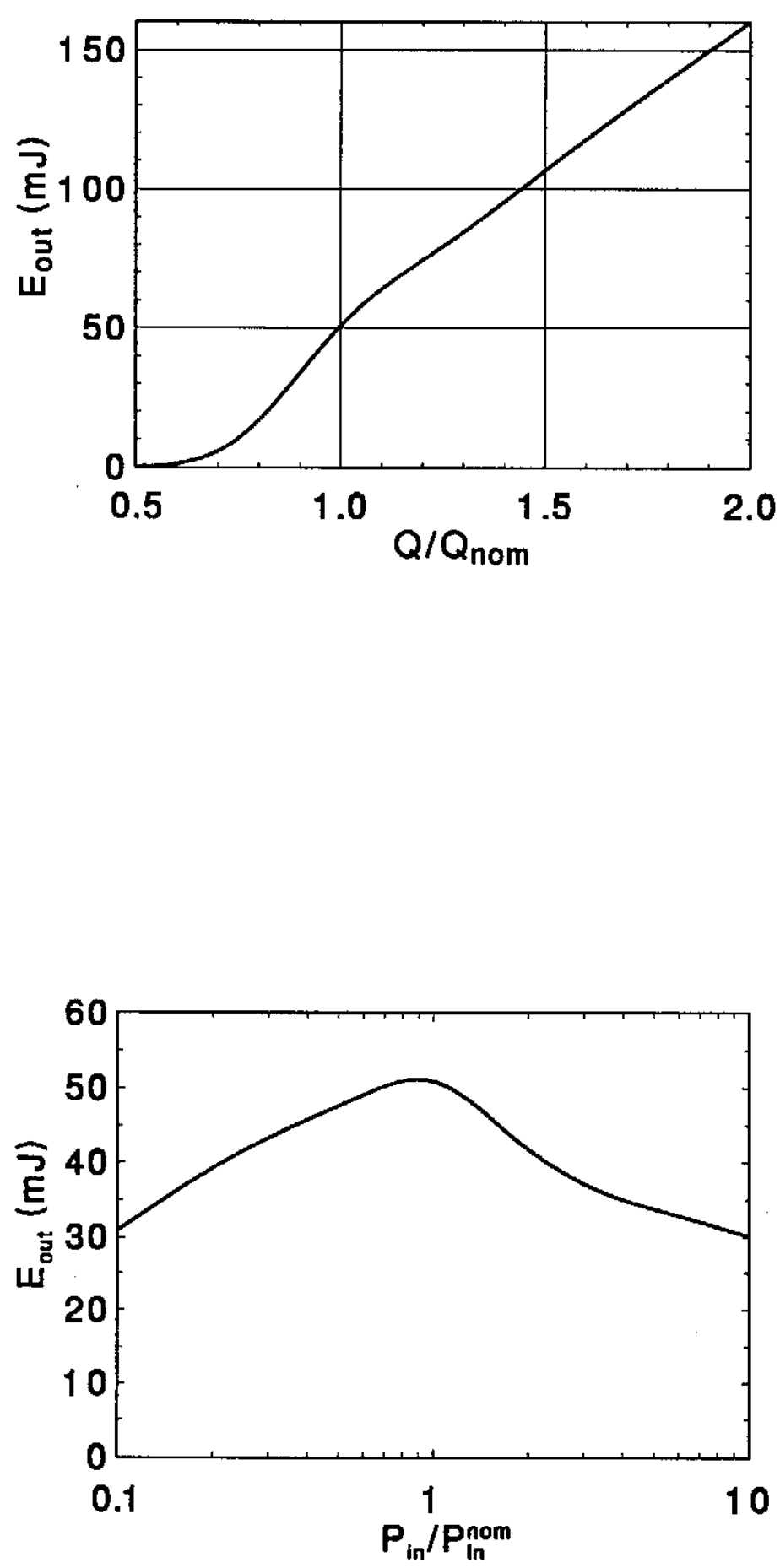

Fig. 16. Dependence of the energy in the radiation pulse on the bunch charge for the FEL amplifier tapered undulator. Total efficiency of the FEL amplifier is equal to $5 \%$
Fig. 17. Dependence of the energy in the radiation pulse on the input radiation power for the FEL amplifier with tapered undulator. Total efficiency of the FEL amplifier is equal to $5 \%$ 
Analysis of the parameters of the UV FEL amplifier (both tapered and untapered options) beyond the project parameters of the TESLA accelerator shows that it will operate reliably even at the value of the emittance exceeding the project value by several times. There is also significant safety margin (by a factor two) with respect to the value of the instantaneous energy spread. So, we can conclude that the ultraviolet free electron laser at the TESLA Test Facility will operate reliably with large safety margins with respect to critical parameters of the accelerator.

\subsection{Cost estimation}

Total cost of the proposed facility consists of three contributions: the master laser, the beam transport line and the undulator. We estimate the cost of the master laser (including optical transport line from the master oscillator of the injection system) to be about $200 \mathrm{kDM}$. The cost of the beam transport line will be also about $200 \mathrm{kDM}$. There are no special technical requirements to the undulator design, so the cost of the undulator will not exceed the value of $100 \mathrm{kDM}$ per meter. As a result, estimated cost of the FEL amplifier with untapered undulator will be about $1700 \mathrm{kDM}$. Upgrade of the facility with additional tapered undulator will cost about $1400 \mathrm{kDM}$.

\section{High intensity gamma-source}

Powerful UV FEL at the TESLA Test Facility can be used for construction of a high intensity, polarized, monochromatic gamma-source at the TESLA Test Facility. The scheme of the gamma-source is presented in Fig. 18. Gamma-quanta are produced in the process of the Compton backscattering of the laser photons on incoming electrons. It has been shown in the previous section that the process of the FEL interaction induces the energy spread in the electron bunch (see Figs. 6 and 12). If the laser radiation will be scattered on the bunches involved in the FEL process, this will result in decreasing of the energy resolution of the gamma-source. To avoid this harmful effect, master laser should operate at a half of the repetition rate of the electron bunches. So, the laser radiation amplified by an electron bunch is focused on the next, unperturbed electron bunch.

Maximal energy of gamma-quanta is defined by the frequency of the primary photons $\omega$ and the energy of the electron $\mathcal{E}:\left(\hbar \omega_{\gamma}\right)_{\max }=\mathcal{E} \chi /(1+\chi)$. Here $\chi=4 \gamma \hbar \omega / m_{\mathrm{e}} c^{2}, m_{\mathrm{e}}$ is the electron mass and $\gamma=\mathcal{E} / m_{\mathrm{e}} c^{2}$ is relativistic factor. Maximal energy of gamma quanta is about $86 \mathrm{MeV}$ at the wavelength of primary photon of $200 \mathrm{~nm}$ and the energy of electron of $1 \mathrm{GeV}$. The intensity of the gamma-source at the TESLA Test Facility can reach the value up to $10^{12}$ gamma-quanta per second which exceed by five orders of magnitude the intensity of existent sources. In principle, the energy of the TTF accelerator can be 


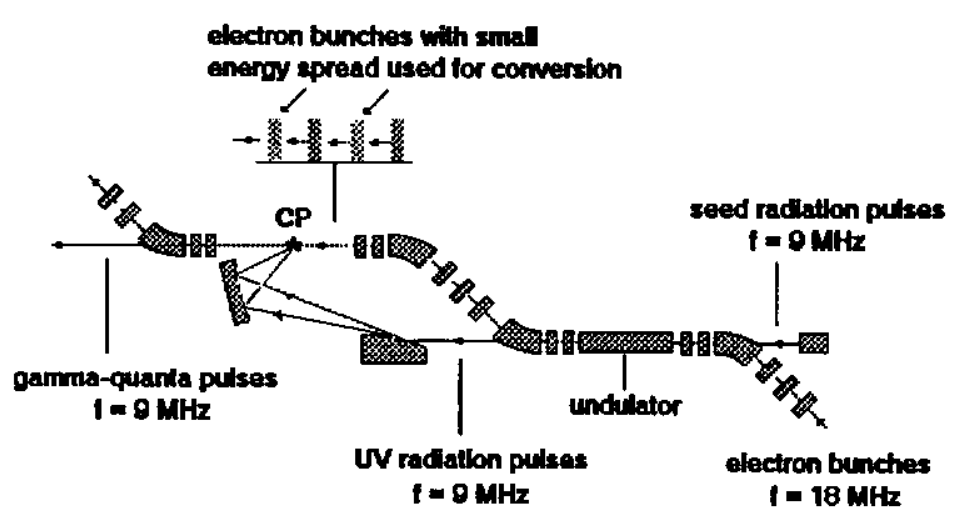

Fig. 18. Scheme of the gamma-source at the TESLA Test Facility

upgraded up to the value of $2 \mathrm{GeV}$. In this case maximal available energy of gammaquanta will be about $320 \mathrm{MeV}$. General parameters of the gamma-source at the TESLA Test Facility are presented in Table 4.

The gamma-source at the TESLA Test Facility can be considered as an ideal one providing an extremely high flux of polarized, monochromatic gamma-quanta. The proposed gamma facility is an extremely selective probe for investigations of not only conventional nuclear physics, but of exotic nuclear states (similar to those which occur inside the core of neutron stars or the quark-gluon plasma) and reaction mechanisms. Up to $200 \mathrm{MeV}$ we are dealing with the internucleon distance about $1 \mathrm{fm}$ (which is of the order of the size of nucleon). Nowadays the investigation of microscopic nuclear structure with resolution $\gtrsim 200 \mathrm{MeV}$ is an unexplored field [9]. The idea is to work in the kinematical region where the processes on free nucleons are forbidden (the cumulative particle production or the underthreshold particle production). This intermediate energy region is covered by nonperturbative QCD effects. Investigations of this transition energy region, from the perturbative to nonperturbative QCD, are the most attractive direction for relativistic nuclear physics to understand the role of the nonnucleonic (pion, $\Delta$, etc, and few-nucleon configurations) and quark-gluon degrees of freedom.

Another interesting topic of these investigations is a possibility to extract additional information about the hadron or the nuclear structure which is usually hidden in the spin-averaged analysis [10]. Polarization observables has a promise of opening a new field in the photoproduction of pion from nucleon and nuclear targets [11], in the processes of photodisintegration of the lightest nuclei $[12,13]$ and others. Many problems in photonucleon and photonulear physics are not resolved till now due to the lack of high quality photon beams.

\subsection{Monochromaticity of gamma-source}

The frequencies of the incident and scattered photons, $\omega$ and $\omega_{\gamma}$, are connected by the relation (in the small-angle approximation): 
Table 4. Parameters of the gamma-source at the TESLA Test Facility

\begin{tabular}{ll}
\hline General parameters & \\
\hline Maximal energy of gamma-quanta & $85 \mathrm{MeV}$ \\
Yield of gamma-quanta & $10^{12} \mathrm{~s}^{-1}$ \\
Energy resolution & $1 \%$ \\
Angular divergence & $0.5 \mathrm{mrad}$ \\
Repetition rate & $10 \mathrm{pps}$ \\
Macropulse duration & $800 \mu \mathrm{s}$ \\
Number of pulses per macropulse & 7200 \\
Micropulse repetition rate & $9 \mathrm{MHz}$ \\
Micropulse duration (FWHM) & $300 \mathrm{fs}$ \\
Parameters of the electron beam & \\
Energy & $1 \mathrm{GeV}$ \\
Beam size at the conversion point (RMS) & $14 \mu \mathrm{m}$ \\
Angular divergence at the conversion point (RMS) & $70 \mu \mathrm{rad}$ \\
Parameters of the laser & \\
\hline Wavelength & $208 \mathrm{~nm}$ \\
Dispersion & Diffraction limited \\
Repetition rate & $10 \mathrm{pps}$ \\
Macropulse duration & $800 \mu \mathrm{s}$ \\
Number of pulses per macropulse & 7200 \\
Micropulse repetition rate & $9 \mathrm{MHz}$ \\
Micropulse duration & $230 \mathrm{fs}$ \\
Energy in the radiation pulse & $50 \mathrm{~mJ}$ \\
Peak output power & $220 \mathrm{GW}$ \\
Average power & $3.6 \mathrm{~kW}$ \\
Parameters of the focusing system & \\
Focuse distance of the mirror & $3 \mathrm{~m}$ \\
Radiation spot size on the mirror & $2.5 \mathrm{~cm}$ \\
Incident radiation flux on the mirror (average) & $350 \mathrm{~W} / \mathrm{cm}{ }^{2}$ \\
Reflectivity of the mirror & $99 \%$ \\
Radiation spot size at the conversion point & $8 \mu \mathrm{m}$ \\
Rayleigh length of the radiation beam & $1 \mathrm{~mm}$ \\
Conversion efficiency & $0.23 \%$ \\
\hline
\end{tabular}

$$
\hbar \omega_{\gamma}=\frac{\mathcal{E} \chi}{1+\chi+\gamma^{2} \theta^{2}},
$$

where $\theta$ is the scattering angle. It is seen from eq. (1) that there is strict correlation between the energy of the backscattered photon and the scattering angle. This allows one to use angle selection technique for selecting gamma-quanta with the required energy. In the case of monoenergetic and laminar electron beam this technique allows, in principle, to achieve any required energy resolution of gamma-quanta. In practice electron beam has finite energy spread which imposes a limit on minimal energy resolution of the gammasource. RMS energy spread in the electron beam of the TTF accelerator is equal to $1 \mathrm{MeV}$, so the lower limit on the energy resolution of the gamma-source is about $0.2 \%$. Influence of the angle spread in the electron beam on the energy resolution of the gamma-source can be eliminated by an appropriate choice of the beta-function at the conversion point. The value of the rms angle spread in the electron beam is given by the expression:

$$
\sigma_{\theta}=\sqrt{\epsilon / \beta},
$$

where $\epsilon$ is the emittance of the electron beam and $\beta$ is beta-function at the conversion point. Minimal energy resolution of the gamma-source is given by the expression: 


$$
\left(\Delta \hbar \omega_{\gamma}\right) / \hbar \omega_{\gamma} \simeq\left(\gamma \sigma_{\theta}\right)^{2} / 2 .
$$

For the case of the electron beam of the TTF accelerator normalized rms emittance is equal to $2 \mathrm{~mm}$ mrad and the energy resolution of gamma-quanta of $1 \%$ is achieved at the value of the beta function of $20 \mathrm{~cm}$ at the conversion point. To achieve ultimate energy resolution, $0.2 \%$, of the gamma-source at the TESLA Test Facility (which is defined by the energy spread of $1 \mathrm{MeV}$ in the electron beam), the value of the beta function at the conversion point should be about $1 \mathrm{~m}$.

\subsection{Polarization of gamma-quanta}

In the present design free electron laser is used as a source of primary photons. FEL radiation is always totally polarized: linearly or circularly for a planar or helical undulator, respectively. In the case of circular polarization of the laser beam, spectral density of backscattered gamma quanta is given with the relation:

$$
\frac{d N^{+}}{d \xi} \propto \xi^{2} \quad \frac{d N^{-}}{d \xi} \propto(1-\xi)^{2}
$$

where $\xi=\hbar \omega_{\gamma} /\left(\hbar \omega_{\gamma}\right)_{\max }$ and notations $N^{+}$and $N^{-}$correspond to the right and left helicity of the $\gamma$-quanta. So, there is correlation of the energy of the gamma quantum and its polarization and the angular selection provides not only monochromatization, but determines also the polarization of gamma quanta (see Fig. 19).

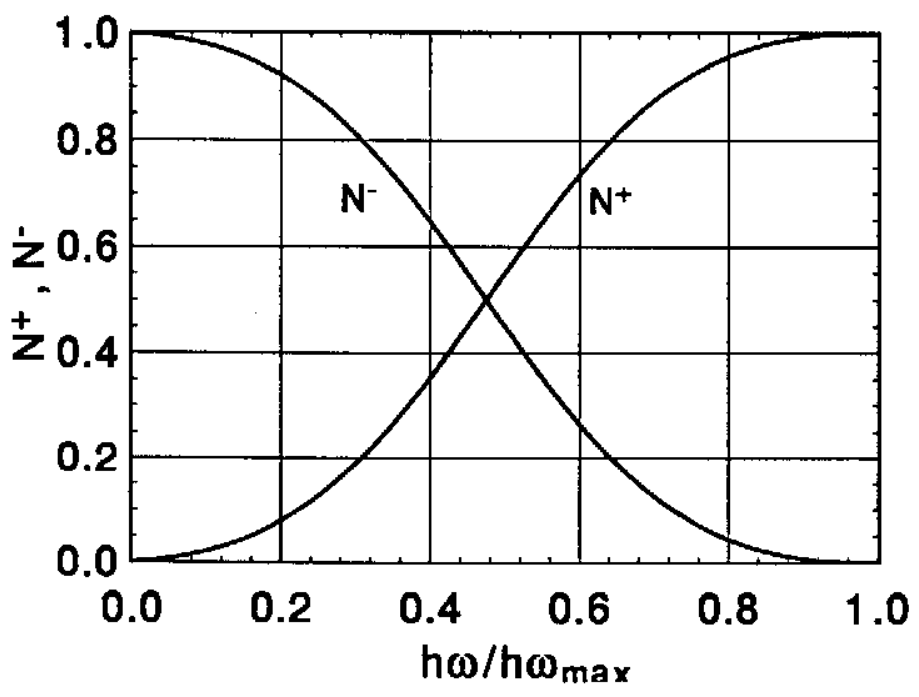

Fig. 19. Spectral density of backscattered $\gamma$-quanta for the case of circularly polarized laser light. Notations $\mathrm{N}^{+}$and $\mathrm{N}^{-}$ correspond to the right and the left helicity of the $\gamma$-quanta

\subsection{Yield of gamma-quanta}

In the general case the yield of gamma-quanta is given by the expression:

$$
d N_{\gamma} / d t=2 c f \sigma_{\mathrm{c}} N_{\mathrm{p}} N_{\mathrm{e}} \int \rho_{\mathrm{p}}(\boldsymbol{r}, t) \rho_{\mathrm{e}}(\boldsymbol{r}, t) d \boldsymbol{r} d t
$$


where

$$
\sigma_{\mathrm{c}}=2 \pi r_{\mathrm{e}}^{2}\left[\frac{1}{\chi} \ln (1+\chi)-\frac{8+4 \chi}{\chi^{3}} \ln (1+\chi)+\frac{8}{\chi^{2}}+\frac{2+\chi}{2(1+\chi)^{2}}\right]
$$

is the total Compton cross section on unpolarized electrons, $r_{\mathrm{e}}=e^{2} / m_{\mathrm{e}} c^{2}, f$ is the repetition rate of the collisions and $N_{(\mathrm{p}, \mathrm{e})} \rho_{(\mathrm{p}, \mathrm{e})}$ are the densities of the photon and the electron beams $\left(\int \rho d r=1\right)$. In the case of the Gaussian distribution of the particles in the electron beam (transverse and longitudinal) we have:

$$
\rho_{\mathrm{e}}(r, z, t)=\left[(2 \pi)^{3 / 2} \sigma_{\mathrm{z}} \sigma_{\mathrm{r}}^{2}(z)\right]^{-1} \exp \left[-\frac{r^{2}}{2 \sigma_{\mathrm{r}}^{2}(z)}-\frac{(z \mp V t)^{2}}{2 \sigma_{\mathrm{z}}^{2}}\right],
$$

where

$$
\sigma_{\mathbf{r}}(z)=\sigma_{\mathbf{r}}(0) \sqrt{1+\frac{z^{2}}{\beta^{2}}}, \quad \sigma_{\mathbf{r}}(0)=\sqrt{\epsilon \beta},
$$

$\epsilon$ is the rms electron beam emittance, $\sigma_{\mathrm{z}}$ is the $\mathrm{rms}$ bunch length and $\beta$ is the beta function at the interaction point.

We assume the laser beam to be close to the Gaussian one. The power flux density in the Gaussian laser beam is given by the expression:

$$
\frac{d P(r, z)}{d S}=\frac{2 W k^{2} w^{2}}{\pi\left(4 z^{2}+k^{2} w^{4}\right)} \exp \left[-\frac{2 k^{2} w^{2} r^{2}}{4 z^{2}+k^{2} w^{4}}\right],
$$

where $W$ is the laser beam power, $w$ is the size of the Gaussian beam waist and $\lambda=2 \pi / k$ is the radiation wavelength. Space density of the photons is described by the expression similar to (7):

$$
\rho_{\mathrm{p}}(r, z, t)=\left[(2 \pi)^{3 / 2} \sigma_{\mathrm{pz}} \sigma_{\mathrm{pr}}^{2}(z)\right]^{-1} \exp \left[-\frac{r^{2}}{2 \sigma_{\mathrm{pr}}^{2}(z)}-\frac{(z \mp c t)^{2}}{2 \sigma_{\mathrm{pz}}^{2}}\right]
$$

where

$$
\sigma_{\mathrm{pr}}(z)=\frac{w}{2} \sqrt{1+\frac{4 z^{2}}{k^{2} w^{4}}}
$$

and $\sigma_{\mathrm{pz}}$ is the rms laser pulse length.

To obtain high yield of gamma-quanta one should provide maximal spatial density of the primary photons and of the electron beam in the conversion region. In the case under study the minimal value of the focusing beta function at the conversion point of about $20 \mathrm{~cm}$ is defined by the required energy resolution of the gamma-source of $1 \%$. Taking into account that the electron and the photon pulse lengths (of about $100 \mu \mathrm{m}$, see Fig. 10) are small with respect to the value of the beta function, we can neglect the longitudinal change of the transverse profile of the electron beam during collision. Assuming the Rayleigh length of the laser beam, $Z_{\mathrm{R}}=\pi w^{2} / \lambda$, to be large with respect to the length of the laser pulse, we can also neglect the change of the transverse size of 
the photon beam during the collision. As a result, we obtain from eq. (5) that the yield of gamma-source is

$$
\frac{d N_{\gamma}}{d t}=f N_{\mathrm{e}} \delta, \quad \delta=\frac{A \sigma_{\mathrm{c}}}{2 \pi \hbar \omega\left(\epsilon \beta+w^{2} / 4\right)},
$$

where $A$ is the energy in the radiation pulse and $\delta$ is the conversion efficiency (number of gamma-quanta produced by one electron).

\subsection{Technical realization}

The scheme of the gamma-source at the TESLA Test Facility is presented in Fig. 18. General parameters of the gamma-source are presented in Table 4. Gamma-quanta are produced in the process of the Compton backscattering of the laser photons on incoming electrons. For the operation of the gamma-source there is no need in a tunable master laser and we assume to use a master laser similar to that of the photoinjector (see Fig. 20). The 4 th or 5 th harmonic of the Nd glass laser $(\lambda=263 \mathrm{~nm}$ and $208 \mathrm{~nm})$ can be used. In this case it is sufficient to install an additional Nd glass amplifier which will amplify the radiation from the master oscillator of the photoinjection system. At the exit of the amplifier the radiation will be converted into 4 th or 5 th harmonic. Peak power of the radiation will be about $5 \mathrm{~kW}$ within $\sim 10 \mathrm{ps}$ pulse duration. The problem of synchronization of the electron and laser pulses will be solved automatically since they use common master oscillator and timing system. Average output power of the master laser for the UV FEL is by an order of magnitude less than the output power of existent laser for the photoinjector. So, construction of the master laser for the FEL amplifier is technically simple and cheap extension of the existent laser facility at the TTF.

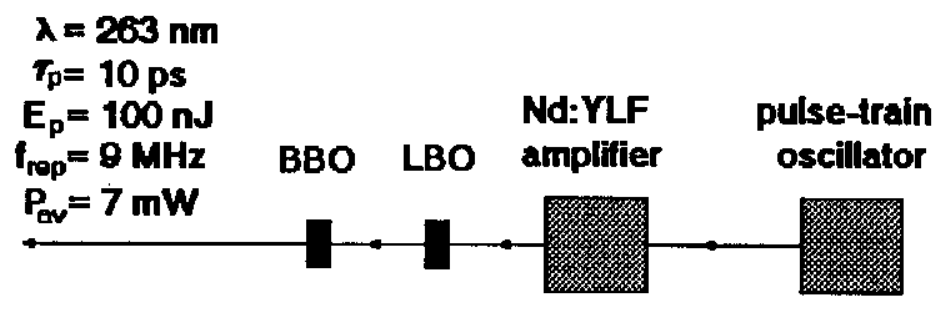

Fig. 20. Scheme of the master laser operating at the 4th harmonic of Nd glass laser

Master laser operates at a half of the repetition rate of the electron bunches and the laser radiation amplified by an electron bunch is focused on the next, unperturbed electron bunch (see Fig. 18). To obtain maximal yield of gamma-quanta, the spatial densities of the photons and of the electrons in the conversion region should be done as high as possible. As it was mentioned above, extra focusing of the electron bunch at the conversion point can reduce energy resolution of the gamma-source due to the increase of the angle spread of the electrons. To provide energy resolution of the gamma-source about of $1 \%$, beta 
function of the electron beam at the conversion point should be about $20 \mathrm{~cm}$. The radiation spot size at the conversion point has been set to $w \simeq 8 \mu \mathrm{m}$ (which corresponds to the Rayleigh length of $1 \mathrm{~mm}$ ). In this case there are no fluctuations of the gamma-source intensity due the time jitter of the electron pulse of about $1 \mathrm{ps.} \mathrm{At} \mathrm{such} \mathrm{a} \mathrm{parameters} \mathrm{of}$ the colliding photon and electron bunches conversion efficiency is about $\delta \simeq 0.23 \%$. At the pulse repetition rate of the accelerator equal to $18 \mathrm{MHz}$ the yield of gamma-quanta is about of $10^{12} \mathrm{~s}^{-1}$.

Ultimate energy resolution of the gamma-source presented in Fig. 18 is limited by the energy spread in the electron beam induced by the SASE FEL process. Even in the case when there is no seed signal at the entrance of the FEL amplifier, the process of the amplification of the radiation is triggered by the shot noise in the electron beam. Effective value of this noise signal is about $30 \mathrm{~W}$. As in the case of seeded FEL, the radiation power grows exponentially with the undulator length. Simulation of this process shows that the induced energy spread due to the SASE FEL process is about $5 \mathrm{MeV}$ at the exit of the undulator [3] which imposes a limit on ultimate energy resolution of the gamma-source of $1 \%$.

There exists another option for organization of the conversion region prior the entrance of the electron beam into the undulator. It is more complicated with respect to the optical system, but possesses several advantages. First, it provides the possibility to double the yield of gamma-quanta, because each electron bunch can be used for producing gammaquanta. Due to a small value of the conversion efficiency this will not destroy the electron beam and it can be used for the amplification of the radiation in the undulator. Second, an ultimate energy resolution of the gamma-source of about $0.2 \%$ can be achieved. This limit is defined by the energy spread in the electron beam of $1 \mathrm{MeV}$. The value of the beta function at the conversion point should be increased up to $1 \mathrm{~m}$. The yield of the gamma-source with ultimate energy resolution of $0.2 \%$ will be about of $2 \times 10^{11} \mathrm{~s}^{-1}$.

\section{Test facility for the positron source}

All the projects of the next generation linear colliders assume to produce positrons by gamma-quanta in a thin target $[14,15,16]$. Gamma-quanta are produced by the spent electron beam in a long wiggler or undulator. For instance, in the TESLA project the $250 \mathrm{GeV}$ electron beam passes a wiggler of $35 \mathrm{~m}$ length producing powerful gammabeam [14]. The mean energy of gamma-quanta is about $25 \mathrm{MeV}$. Positrons produced in a thin target have large energy and angle spread. After the target they should be captured and accelerated. Such a complicated system requires experimental verification prior construction of the full-scale facility. Such a test facility can be constructed on the 
base of a high intensity gamma-source described in the previous section. Energy spectrum of the gamma-source is similar to that to be used in a full scale facility. Intensity of the gamma-source described above has been limited by the requirement of the energy resolution. For the positron source test facility the value of the beta function at the conversion point can be reduced down to the value of about $1 \mathrm{~cm}$ (the angle divergence in the beam is about $1 / \gamma$ in this case). As a result, the yield of gamma-quanta reaches the value of $10^{13} \mathrm{~s}^{-1}$. Remembering that the conversion efficiency of gamma-quanta into electron-positron pair is about of 0.5 at the thickness of the target about one radiation length, we estimate the yield of the positrons to be about $5 \times 10^{12} \mathrm{~s}^{-1}$. Such a positron yield is sufficient for operation of the test facility. Taking into account that TTF accelerator has the same time diagram of operation as the TESLA accelerator, this facility will allow to verify experimentally all the systems of the positron source (optimization of the target, capture efficiency, etc). The only difference is in a lower heat load of the target, but this problem can be investigated independently, for instance, with powerful electron beam.

The test facility for the positron source could reveal unique opportunity for experimental investigation of the polarized positron source proposed in ref. [17]. The idea of proposal [17] is to produce longitudinally polarized positrons in a thin heavy material target. Circularly polarized primary photons will be generated by a spent electron beam in the undulator. Verification of the technical ideas of the polarized positron source can be done directly with the gamma-source described above. In this case FEL amplifier should be equipped with a helical undulator. Spectral and polarization properties of the gammasource will be identical to those used in the project of the polarized positron source. As it has been mentioned above, the yield of positrons will be sufficient for testing all the accelerator systems.

\section{Test facility for the gamma-gamma collider}

An option of a gamma-gamma collider is included in several projects of the future generation linear colliders $[14,15]$. The scheme for organization of gamma-gamma collisions is presented in Fig. 21. Two electron bunches are focused into the interaction point. Prior the collision each electron bunch is irradiated by a powerful laser pulse. High energy gammaquanta are produced in the process of Compton backscattering and, following the electron trajectories, collide at the interaction region. To provide high conversion efficiency, the peak power of a laser must be rather high. For instance, to achieve $70 \%$ conversion efficiency, the peak laser power should be of about $300 \mathrm{GW}$. The time structure of the laser pulses should be identical to the time structure of the electron bunches. It means, that the laser should have a capability of precise synchronization with the electron bunches (with 
the jitter of about $1 \mathrm{ps)}$ and should provide a high repetition rate. The last parameter is extremely severe for the TESLA collider requiring a TW level peak power laser with the repetition rate of about $1 \mathrm{MHz}$. To provide a more wide range of physical experiments, there should be a possibility to steer the polarization of the colliding gamma quanta which assumes a possibility to steer the polarization of laser light.

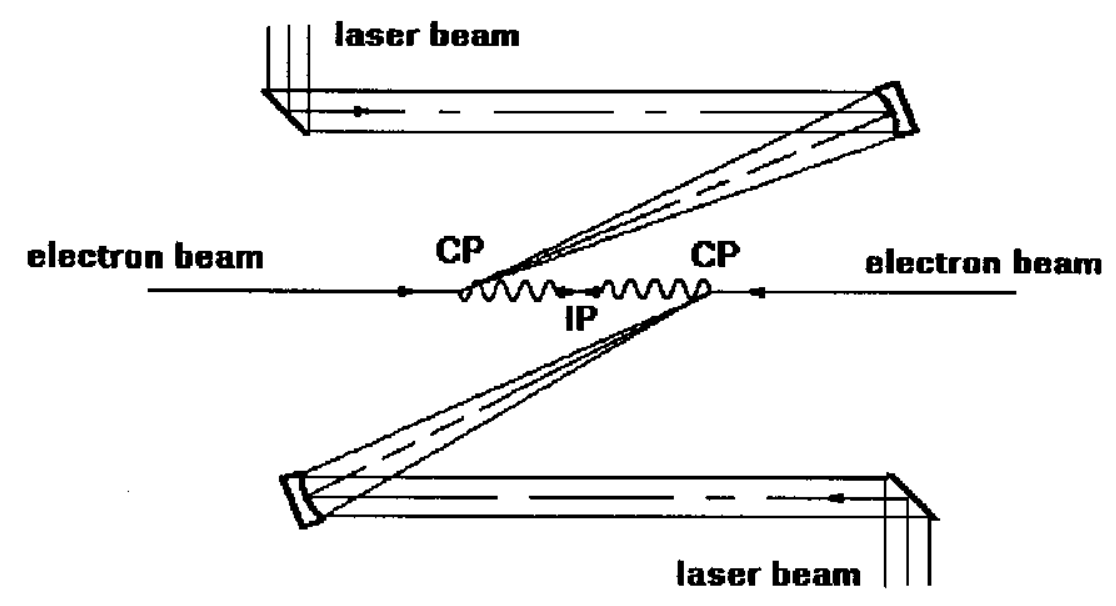

Fig. 21. Scheme of gammagamma collider

In the case of the gamma-gamma collider at TESLA there is no reliable technical solution for a conventional laser system with required parameters [18], and free electron laser system is considered now as a candidate for the laser [14]. Parameters of the high power UV FEL at the TESLA Test Facility and of the optical system of the gamma-source are very close to those used in the project of the gamma-gamma collider. Indeed, peak and average laser power of $220 \mathrm{GW}$ and $7 \mathrm{~kW}$, respectively, are close to those required for the gamma-collider. Time diagram of the laser pulses is similar to the time diagram of the TESLA collider. The problems connected with alignment of the mirrors, possible radiation damage of the mirrors, time jitters and the problems of focusing the laser beam in a tiny spot are similar to those to be met at a full-scale facility. So, successful realization of this project will serve as an experimental verification of a technical feasibility of the gamma-gamma option at TESLA.

\section{Potential for industrial applications}

In conclusion to this paper we should like to attract an attention to possible industrial applications of powerful free electron laser at the TESLA Test Facility. During last few years the problem of industrial applications of a $\mathrm{kW}$ level power FELs intensively discussed in the FEL community. Recently this discussions have been realized in a project of industrial FEL which is supported by a consortium of industrial firms including DuPont, $3 \mathrm{M}$, Xerox, AT\&T and IBM [19]. It is interesting to notice that this project is also based 
on a superconducting accelerator. The chosen FEL configuration is an oscillator. Since this device has low efficiency, the designers of the project have chosen the way of electron recuperation in order to increase the net efficiency of the laser. Another significant problem to be solved during realization of this project is that of the optical resonator connected with a high level of the intracavity power.

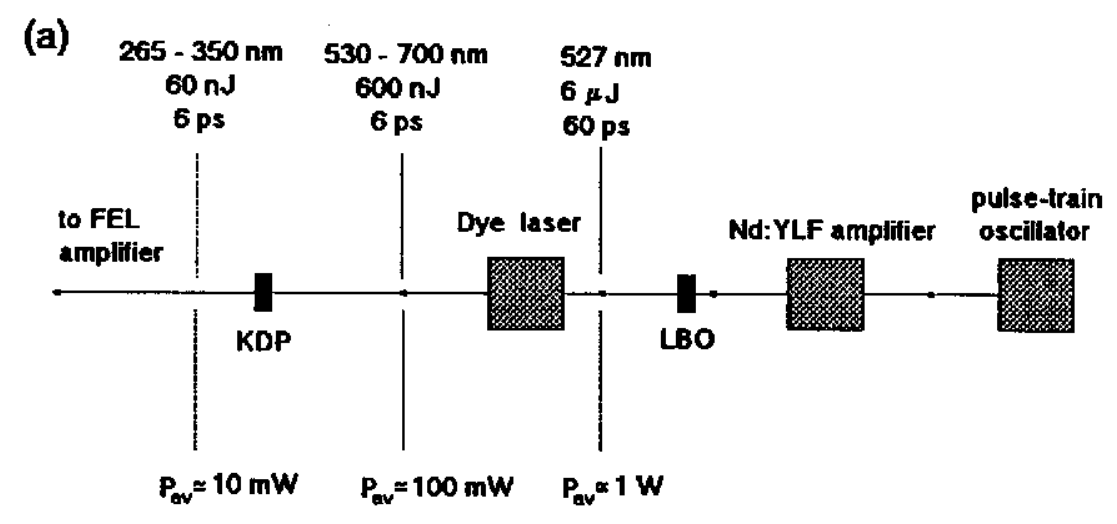

(b)

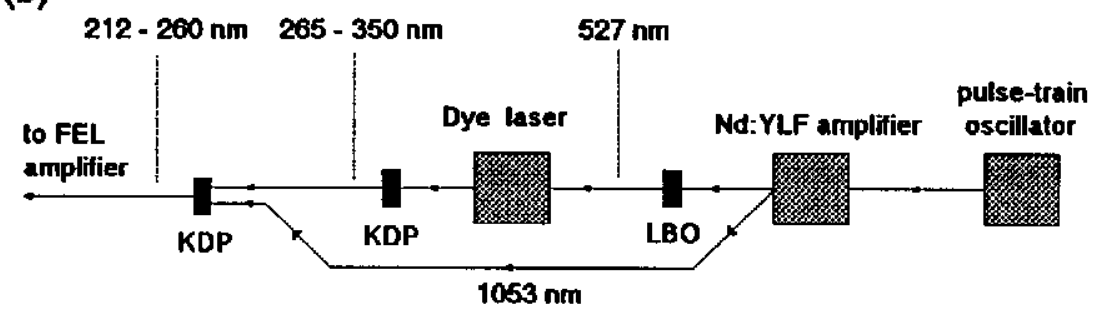

Fig. 22. Schemes of the master laser providing permanent tuning in the Wavelength ranges of $265-350 \mathrm{~nm}$ (plot (a)) and of $212-260 \mathrm{~nm}$ (plot(b))

In this paper we point at the possibility of using an alternative approach for constructing a high power UV FEL. It consists in using a Master Oscillator - Power Amplifier (MOPA) scheme. The problem of a tunable master oscillator can be solved with available dye laser systems pumped by $\mathrm{Nd}$ glass with subsequent using nonlinear optical elements (see Fig. 22). This technique covers wavelength range from $212 \mathrm{~nm}$ to $350 \mathrm{~nm}$. Required level of output average and peak power of about $10 \mathrm{~mW}$ and $10 \mathrm{~kW}$, respectively, can been routinely provided with available laser technique.

A high efficiency FEL amplifier with tapered undulator allows one to overcome significantly the level of the peak and the average output power attainable in an FEL oscillator. This is due to the fact that there are no problems with the mirrors in the high gain FEL amplifier - the process of amplification is performed during a single pass of the radiation through the undulator. Also, there is no limitation on the way of decreasing the operating wavelength or expanding it beyond the harmonics of the dye laser band. Low powerful FEL oscillator can be used in this case as a master laser. Comparison with CEBAF project (see Fig. 23 and ref. [19]) indicates that potential of the UV FEL at the TESLA Test Facility for possible industrial applications should be studied in detail, too. 


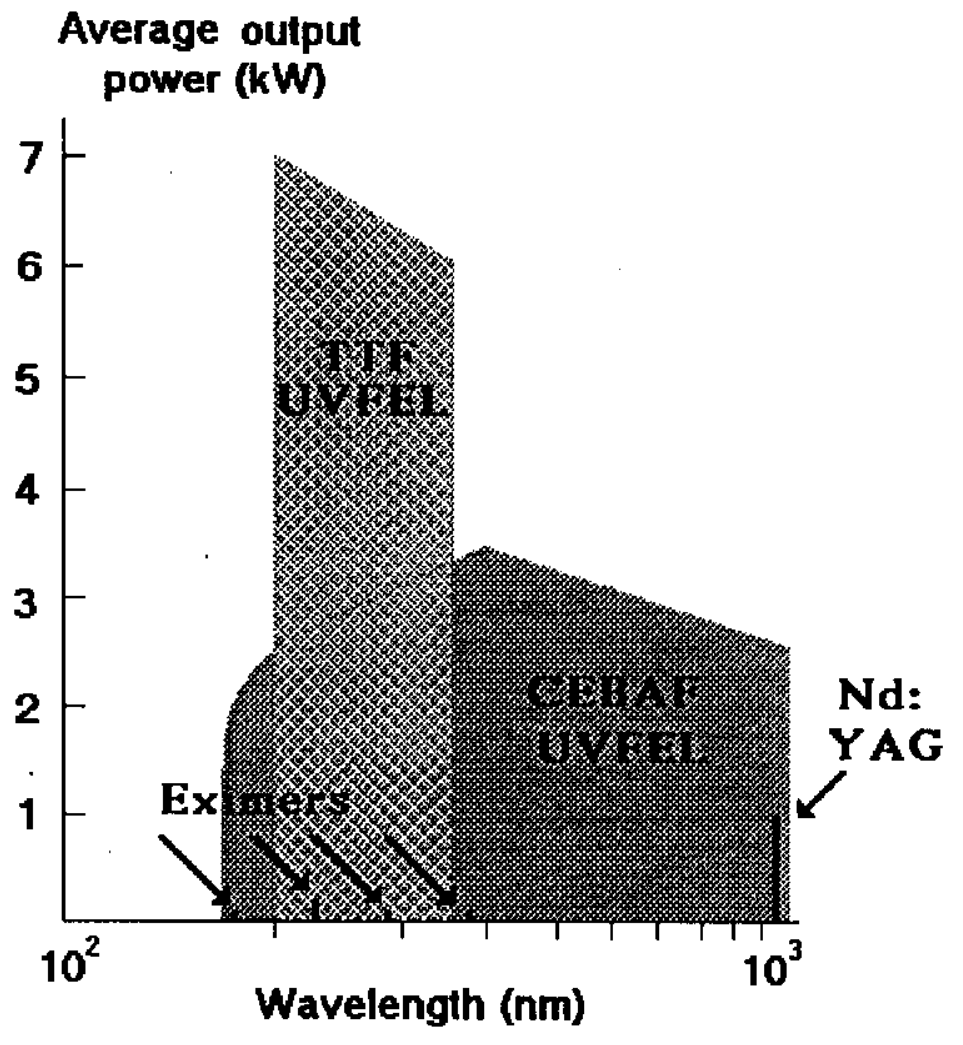

Fig. 23. Operating ranges of the CEBAF UV FEL and of the UV FEL at the TESLA Test Facility

\section{Acknowledgments}

We wish to thank R. Brinkmann, S. Choroba, B. Dwersteg, J. Feldhaus, K. Flöttman, A. Gamp, P. Gürtler, J. Krzywinski, G. Materlik, T. Möller, J. Pflüger, J. Roßbach, J.R. Schneider, S. Schreiber, B. Sonntag, I. Will and J. Weisend II for many useful discussions. 


\section{References}

1. "A VUV Free Electron Laser at the TESLA Test Facility: Conceptual Design Report", DESY Print TESLAFEL 95-03, Hamburg, DESY, 1995.

2. J. Rossbach, Nucl. Instrum. and Methods A375(1996)269.

3. E.L. Saldin, E.A. Schneidmiller and M.V. Yurkov, "Three dimensional, time-dependent FEL simulation code S\&D-3T"

4. E.L. Saldin, E.A. Schneidmiller and M.V. Yurkov, Phys. Rep. 260(1995)187.

5. N. Kroll, P. Morton and M. Rosenbluth, SRI Rep. JSR-79-01 IEEE J. Quantum Electron. QE-17(1981)1436.

6. C.M. Tang and P. Sprangle, Phys. Quantum Electron. 9(1982)627.

7. D. Prosnitz, A. Szoke and V.K. Neile, Phys. Rev. A 24(1981)1436.

8. T.J. Orzechowski et al., Phys. Rev. Lett. 57(1986)2172.

9. L. Frankfurt, SLAC Workshop on High Energy Electroproduction and Spin Physics, SLAC-392 Conf-920266 UC-414, 1992.

10. R.P. Feynman, Photon-hadron interactions (W.A. Benjamin, Inc., 1972).

11. A.A. Chumbalov et al.,Phys. Letters, B321(1994), 317-322; D. Drechsel, MKPH-T-93-9, Mainz, 1993.

12. V. Isbert et al., DAPNIA/SPhN 93 64, C.E. Saclay, 1993

13. H, Arenhövel and M. Sanzone, "Photodisintegration of the Deuteron", Springer-Verlag Wien - New York, 1991

14. "Conceptual Design of a $500 \mathrm{GeV}$ e+e- Linear Collider with Integrated X-ray Laser Facility" (Editors R.Brinkmann, G. Materlik, J. Rossbach, A. Wagner), DESY 97-048, Hamburg, 1997.

15. "Zeroth-Order Design Report for the Next Linear Collider", LBNL-PUB-5424,SLAC Report 474, UCRL-ID124161 (May 1996).

16. Status reports on CLIC, JLC and VLEPP are given in: Proceedings of the LC'92 ECFA. Workshop on $e^{+} e^{-}$ Linear Colliders (July,25-August,2,1993,Germany), MPI-PhE/93-14, ECFA 93-154.

17. V.E. Balakin and A.A. Mikhailichenko, Proc. of the 12th Int. Conference on High Energy Accelerators (Batavia, 1983), p. 127.

Preprint INP 79-85, Novosibirsk, 1979.

18. E.L. Saldin, V.P. Sarantsev, E.A. Schneidmiller, Yu.N. Ulyanov and M.V. Yurkov, Nucl. Instrum. and Methods A.361(1995) 101.

19. G.R. Neil, S.V. Benson, H.F. Dylla and H. Liu, Nucl. Instrum. and Methods A358(1995)159. 